\title{
Paraneoplastic Opsoclonus Ataxia
}

National Cancer Institute

\section{Source}

National Cancer Institute. Paraneoplastic Opsoclonus Ataxia. NCI Thesaurus. Code C8966.

Ataxia associated with chaotic multidirectional conjug ate eye movements secondary to metastatic tumors to cerebellum. The most common malignant tumor associated with this paraneoplastic syndrome is lung carcinoma. $(\mathrm{NCl})$ 